\title{
Stress distribution in conical sandpiles formed with ellipsoidal particles
}

\author{
Sida Liu, Zongyan Zhou*, Ruiping Zou and Aibing Yu \\ Laboratory for Simulation and Modelling of Particulate Systems, Department of Chemical Engineering, Monash University, \\ Melbourne, VIC 3800, Australia
}

\begin{abstract}
The properties of a sandpile such as angle of repose and stress distribution are affected by many variables, among which particle shape is one of the most important ones. In this work, ellipsoids which can represent a large range of shapes varying from disk- to cylinder-type are used. The discrete element method (DEM) is employed in order to conduct controlled numerical experiments. The results show that $3 \mathrm{D}$ conical sandpiles have similar properties as $2 \mathrm{D}$ ones reported in the literature. It demonstrates that particle shape affects the magnitude of the contact force network significantly, with spheres being the smallest. As expected, the pressure distribution underneath sandpiles is featured with a relatively small pressure in the centre, and ellipsoids have a more significant stress dip region than spherical particles.
\end{abstract}

\section{Introduction}

A sandpile is one of the most typical particulate systems which are widely encountered in nature and many industries. It has two important properties including angle of repose and stress distribution, which have been investigated extensively in the literature. For example, angle of repose, as one of the most important parameters in characterizing the behavior of granular materials, is closely related to many phenomena, including packing, avalanching, stratification and segregation. Stress distribution is another interesting feature of granular materials which can affect many practical operations from silos and building foundations to road and dam construction, hence it is important to understand the physics underlying piling processes.

In the past decades, the two properties have been studied experimentally or mathematically. In particular, the so called discrete element method (DEM) [1], as a dynamic simulation technique, can take into account all dynamic factors related to either geometry or forces, hence it has been widely used to study sandpiles, as summarized by Zhu et al. [2]. Generally speaking, the shape and size of sandpiles are a macroscopic result from collected effects of various internal mechanisms involving many physical variables. For example, the magnitude of the angle of repose depends on pile formation method, and a range of other variables related to material properties such as sliding friction coefficient, rolling friction coefficient, density of particles, and particle characteristics such as size and shape. The stress distribution in a sandpile, particularly the pressure dip phenomenon, is often examined. It is known that the pressure distribution beneath a sandpile is affected by various factors, including formation scheme, particle properties, flow rate, pouring height, and bottom geometry. Among these factors, particle shape is an important parameter affecting the two properties, as illustrated by some investigators [3-7].

Ellipsoid is an ideal particle shape that can represent a wide range of shapes varying from disk like to cylinder like particles. Its shape for spheroids can be described by one single parameter, i.e. aspect ratio. Also, the surface of ellipsoids can be accurately described by the quadratic equations, and can be handled readily by DEM. Thus, ellipsoids have been increasingly used to examine the effect of particle shape on different behaviour of granular materials, for example, as demonstrated in recent studies of packing [8], fluidization [7] and hopper flow [9]. In the previous study, the effect of particle shape on sandpile properties was investigated in a $2 \mathrm{D}$ slot model [10]. It showed that ellipsoids have more pronounced stress dip than spheres, and the pressure distribution underneath sandpiles is featured a relatively constant normal pressure in the central region rather than a dip. It is not clear how the model geometry affects the two sandpile properties. In the present study, particle shape effect is carried out in a full 3D conical sandpile, and the results are compared with 2D slot models.

\section{DEM for ellipsoids}

DEM for ellipsoidal particles have been reported in the literature [8]. The governing equations can be written as:

$$
m_{i} \frac{d \mathbf{v}_{i}}{d t}=\sum_{j=1}^{k_{c}}\left(\mathbf{f}_{c, i j}+\mathbf{f}_{d, i j}+\mathbf{f}_{v, i j}\right)+m_{i} \mathbf{g}
$$

\footnotetext{
Corresponding author: zongyan.zhou@monash.edu
} 
and

$$
I_{i} \frac{d \boldsymbol{\omega}_{i}}{d t}=\sum_{j=1}^{k_{c}}\left(\mathbf{M}_{t, i j}+\mathbf{M}_{r, i j}+\mathbf{M}_{n, i j}\right)
$$

where $v_{i}$ and $\omega_{i}$ are the translational and angular velocities of the particle, respectively, and $k_{c}$ is the number of particles in interaction with the particle. The forces involved are: the gravitational force $\mathrm{m}_{\mathrm{i}} \mathrm{g}$, and inter-particle forces between particles, which include elastic force $f_{c, i j}$, and viscous damping force $f_{d, i j}$. For fine particles, the van der Waals force $f_{v, i j}$ needs to be considered. These inter-particle forces can be resolved into the normal and tangential components at a contact point. The torque acting on particle $\mathrm{i}$ by particle $\mathrm{j}$ includes two components: $\mathrm{M}_{\mathrm{t}, \mathrm{ij}}$ which is generated by the tangential force and causes particle $\mathrm{i}$ to rotate, and $\mathrm{M}_{\mathrm{r}, \mathrm{ij}}$, commonly known as the rolling friction torque, is generated by asymmetric normal forces and slows down the relative rotation between particles. For ellipsoids, addition torque $\mathrm{M}_{\mathrm{n}, \mathrm{ij}}$ should be added because the normal contact force and van der Waals force do not necessarily pass through the particle centre. Other critical features for ellipsoids are introduced briefly below:

- Determining the interaction forces by calculating the radius $R^{*}$, which is strongly related to the radii of the curvature at a contact point;

- Using geometric potential algorithm to detect the contacts between ellipsoids; and

- Particle orientation is defined by three Euler angles $(\phi$, $\Theta, \Psi$ ) based on the quaternion method.

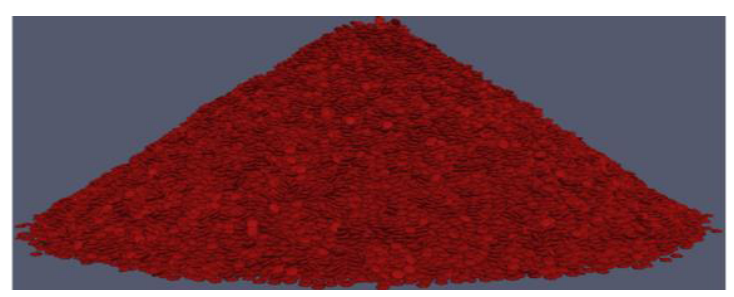

(a)

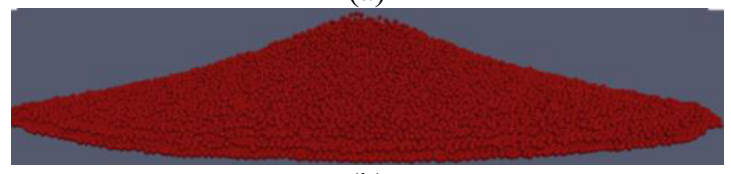

(b)

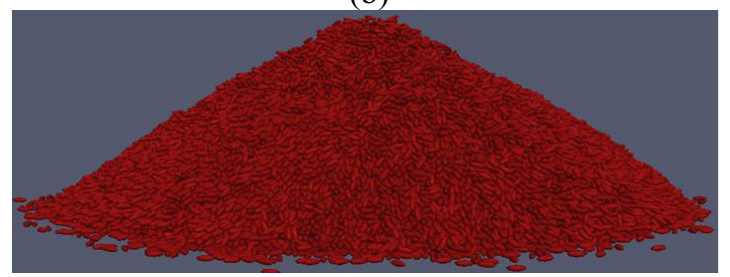

(c)

Fig. 1. 3D conical sandpiles formed for different aspect ratios of ellipsoids: (a) 0.3, (b) 1.0, and (c) 3.0.

\section{Simulation conditions}

A packing is formed first in a cylindrical hopper above a horizon plate, and an orifice is located in the central flat bottom. 80,000 mono-sized particles are poured into the hopper and allowed to settle under gravity. The orifice is then opened thus let particles discharge from the hopper. The instantaneous opening of the outlet starts a discharging process in which particles drop onto the bottom surface under gravity, and form a sandpile beneath the hopper. The distance between the orifice and the bottom plate is set to $0.5 \mathrm{~m}$, and there are no side wall boundaries for the bottom surface. The formation of full 3D conical sandpiles are shown in Fig. 1.

Note that each ellipsoid has three diameters in three principal directions, where $a$ is the principal radius in the polar direction, $\mathrm{b}$ and $\mathrm{c}$ are principal radii in the equatorial plane. For simplification, b is equal to c, resulting in all particles being in the class of spheroids. Hence, the aspect ratio is defined as the ratio of $a$ to $b$ $(=c)$. Obviously, for a prolate spheroid, aspect ratio $>1$; for a sphere, aspect ratio $=1$; and for an oblate spheroid, aspect ratio $<1$. Spheroids with aspect ratio varying from 0.3 to 3.0 are used in this work to produce different shapes. Detailed parameters used in the DEM simulation are listed in Table 1.

Table 1. Parameters used in DEM simulation.

\begin{tabular}{|l|l|}
\hline Quantity & \multicolumn{1}{|c|}{ Values } \\
\hline Number of particles & 80,000 \\
\hline Dropping height & $0.5 \mathrm{~m}$ \\
\hline Sliding friction coefficient $(\mathrm{p}-\mathrm{p})$ & 0.4 \\
\hline Sliding friction coefficient $(\mathrm{p}-\mathrm{w})$ & 0.4 \\
\hline Young's modulus & $1 \times 10^{7} \mathrm{~Pa}$ \\
\hline Damping coefficient & 0.3 \\
\hline Time step & $3.2 \times 10^{-5} \mathrm{~s}$ \\
\hline Aspect ratio & $0.3 \sim 3.0$ \\
\hline Particle size $\left(2 \mathrm{a}, 2 \mathrm{~b}=2 \mathrm{c}, \mathrm{d}_{\mathrm{p}}\right)$ & $\mathrm{d}_{\mathrm{p}}=2(\mathrm{abc})^{1 / 3}=0.01 \mathrm{~m}$ \\
\hline
\end{tabular}

\section{Results and discussion}

\subsection{Angle of repose}

Fig. 2 shows the variation of angle of repose with aspect ratio as well as orifice size. Note that a $3 \mathrm{D}$ stockpile could have slightly different angle of repose in different directions, thus the angle of repose measured in the present study is an average value of 8 directions. Spherical particles have the lowest angle of repose as observed in the literature [10]. For oblate particles, the angle of repose increases with aspect ratio deviating from sphere and reach a maximum value at aspect ratio 0.5 , then decrease. For prolate particles, the angle of repose keeps increasing with aspect ratio but the rate of slope is decreased for aspect ratio larger than 1.5. This curve is similar to the variation of coordination number with aspect ratio for particle packing [8]. The orifice size of hopper flow in the upper part can make effect on angle of repose as well. Small orifice size usually leads to relatively large angle of repose.

\subsection{Force structure}

The contact forces between particles distribute nonuniformly and form a complex force network in a 
granular system. This has been confirmed by previous experimental studies in two dimensions and DEM simulation in a 2D slot model [10]. Fig. 3 shows the force networks for different aspect ratios. The forces between particles are demonstrated by the thickness and colors that are proportional to the magnitude of the normal contact force (red represents large forces and blue represents small forces). It can be observed that the large contact forces mainly propagate from the base toward the top of the sandpile for all the cases. Spheres have the smallest angle of repose thus the force network is weak correspondingly. With aspect ratio deviating from 1.0, the height of sandpiles increases and forms a relatively strong force network. The force network observed is similar to the slot models in reference [10].

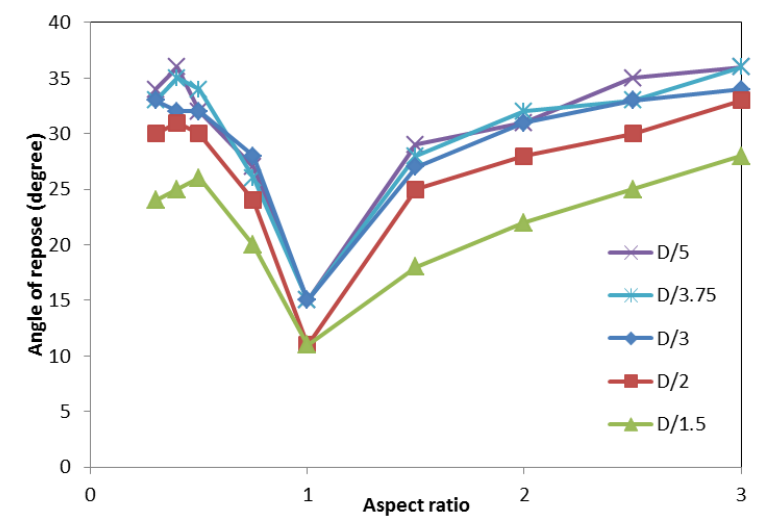

Fig. 2. Average angle of repose of sandpiles formed for different aspect ratios and discharge rate.

0.3

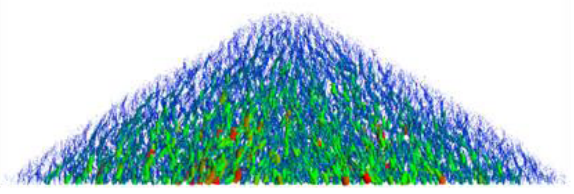

0.5

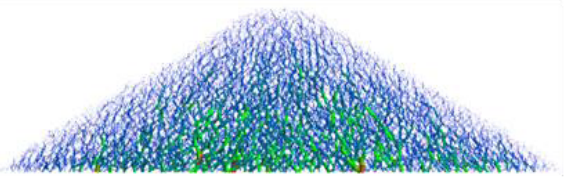

1.0

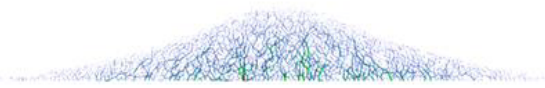

1.5

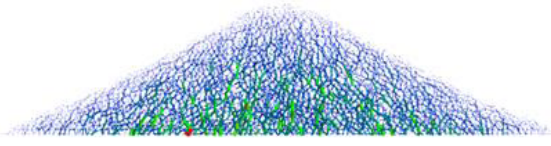

2.5

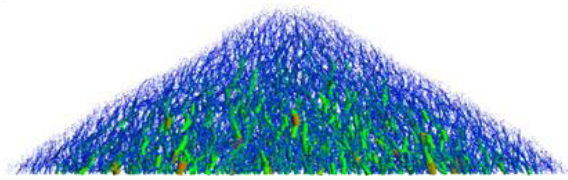

Fig. 3. Force structure in the central slice of sandpiles formed by particles of different aspect ratios.

One particular feature in the force network is the contact force orientation. Fig. 4 shows the normal contact force orientation of ellipsoids for different aspect ratios. For oblate spheroids, a strong peak can be observed at low angle. This is mainly due to particle orientation distribution that most particles are facing upward or downward, results in large number of vertical forces. As aspect ratio approaches 1.0 (spheres), the forces directions become more uniform. A hump is observed instead of peaks. The highest point of the hump shows up at about 45 degrees and another high point is at 90 degrees. This indicates a large number of contact forces are in random directions for spheres. For prolate particles, the distribution is similar to oblate particles, but the peak is not significant. This is because most prolate particles are lying horizontally.

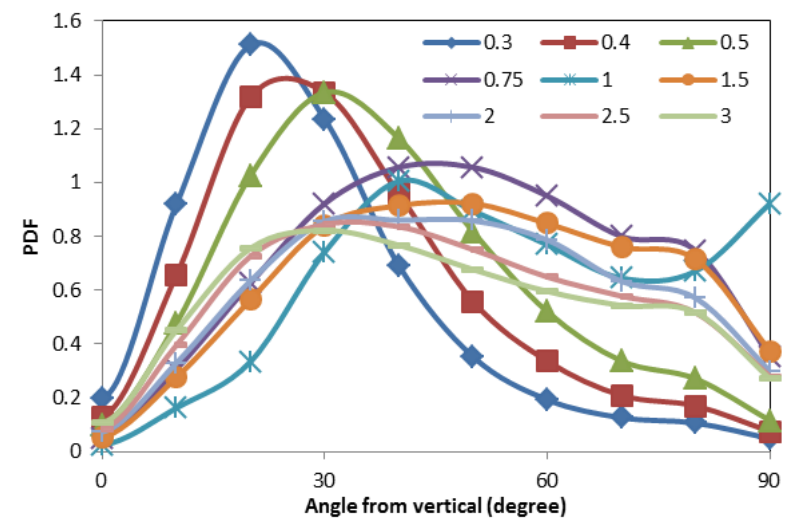

Fig. 4. Static of normal contact force directions of a sandpile for different aspect ratios.

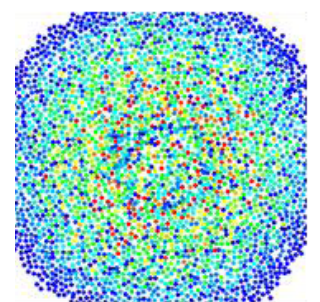

0.3

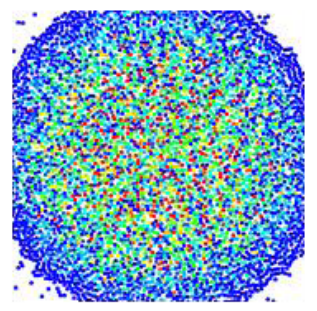

2.0

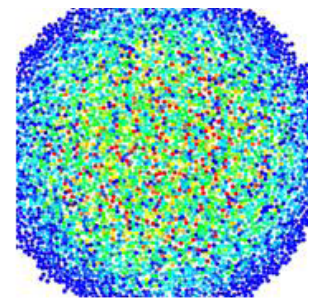

0.5

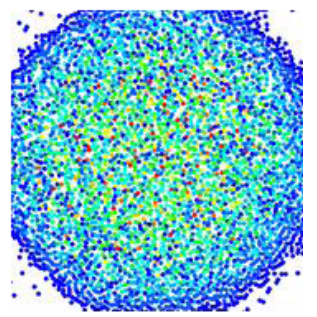

3.0
Fig. 5. Normal force distribution on bottom plate for different aspect ratios.

\subsection{Bottom stress}

Fig. 5 shows the normal force distribution on the bottom plate for different aspect ratios. Each point in the figure represents a contact point between particle and bottom plate, and its color reflects the magnitude of the force (red represents large forces and blue represents small forces). For some cases (e.g., aspect ratio of 0.3), relatively small forces can be observed in the central region and most large forces are distributed around the centre. For other cases, forces with various magnitudes are spread in a wide range, and it is hard to describe force distribution on the basis of these patterns. 


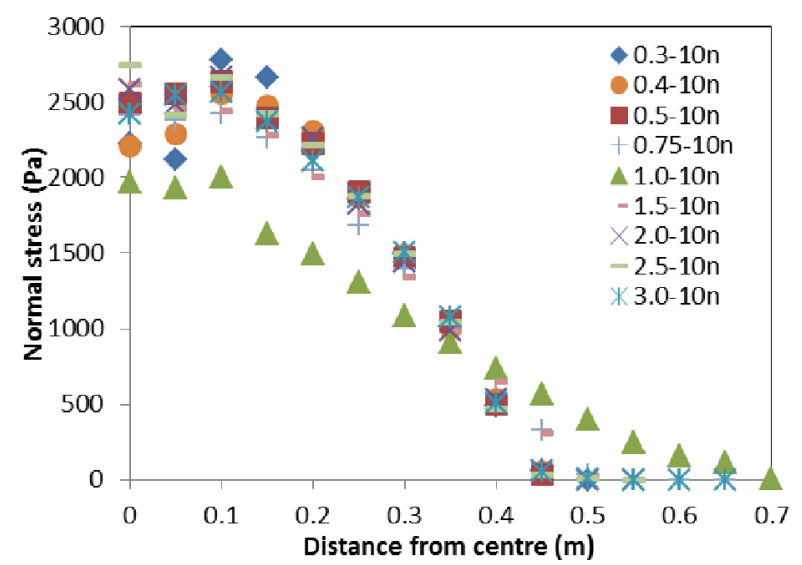

(a)

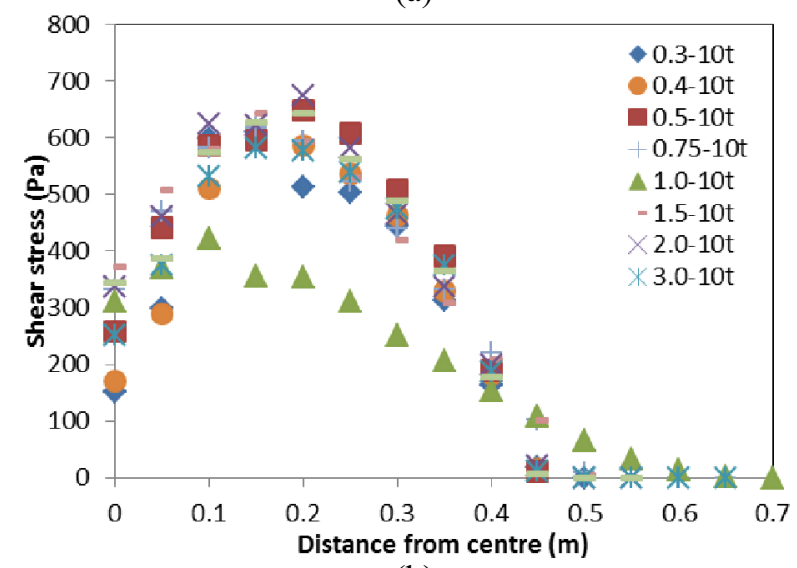

(b)

Fig. 6. Average bottom stress distribution for (a) normal stress and (b) shear stress.

It is often observed that a stress dip occurs beneath a sandpile. However, there is no consistency on this phenomenon as reported in the literature. Generally, it is known that the pressure distribution beneath a sandpile is affected by many factors, such as construction history, base deflection, particle size distribution and particle friction. Fig. 6 shows the normal and tangential stress beneath the sandpiles for different aspect ratios when orifice is $0.1 \mathrm{~m}$. To illustrate the stress dip distribution, the pressure is calculated using a ring that is $0.05 \mathrm{~m}$ in width to obtain a relatively reliable value. For tangential stress, the stress dip can be observed for all the cases considered. But for normal stress, the dip is not so clear, consistent with the results as observed in the $2 \mathrm{D}$ slot model [10]. These results of stress distribution can generally be divided into three groups. The first group is stress dip group, for example, aspect ratio at 0.3 and 0.4 , the stress dip phenomenon is very clear.The second group is terrace-like group, such as aspect ratio at 0.75 and 1.0. The stress in the middle section is tending to be uniform and form a terrace-like curve. The third group is stress rise group, such as aspect ratio at 1.5 and 2.5. For these cases, instead of a dip, the stress in the centre is higher than other areas, and the dip is observed at a small distance from the centre.

\section{Further discussions and conclusion}

The angle of repose obtained from sandpiles formed by ellipsoidal particles is comparable with previous studies. Spherical particles can have the smallest angle of repose and the trend for oblate and prolate spheroids differs. For oblate spheroids with aspect ratio deviating from sphere, the angle of repose firstly increases and the peak value is obtained when aspect ratio is 0.5 , then the angle of repose will drop. For prolate spheroids, the angle of repose is larger than spheres but the increase rate is low for large aspect ratios. The trend is similar to average coordination number of packing of ellipsoids. The larger orifice size results in the smaller angle of repose.

Stress distribution is analysed to investigate the effect of particle shape and orifice size. Force distribution inside a sandpile is significantly related to aspect ratio. Vertical forces are more likely to be observed for ellipsoids, whilst large number of horizontal forces is obtained for spheres. Bottom force distribution also differs with aspect ratio. Stress dip can always be obtained for tangential stress. But for normal stress, certain cases shows a flat terrace in the centre (sphere), certain cases show stress dip (oblate spheroids with aspect ratio at 0.4 ), and certain cases show stress rise (prolate spheroids with aspect ratio of 2.5). There is no clear relationship between aspect ratio and stress dip phenomenon. But compare with previous 2D slot model studies, stress dip is more likely to obtain in the 3D DEM simulation as shown in the present study. Orifice is another factor can influence bottom stress. Most particle shapes can have stress dip when orifice is small, and enlarged orifice size leads to terrace-like or stress rise distribution.

\section{References}

1. Cundall, P.A. and O.D.L. Strack, Geotechnique, 29, 1, (1979).

2. Zhu, H.P., et al., Chemical Engineering Science, 63, 23, (2008).

3. Zhou, C. and J.Y. Ooi, Mechanics of Materials, 41, 6, (2009).

4. Zuriguel, I. and T. Mullin, Proceedings of The Royal Society A, 464, 2089, (2008).

5. Ai, J., et al., Granular Matter, 13, 2, (2011).

6. Liu, J.G., Q.C. Sun, and F. Jin, Powder Technology, 212, 1, (2011).

7. Zhu, J.Y., Y.Y. Liang, and Y.H. Zhou, Powder Technology, 234, 0, (2013).

8. Zhou, Z.Y., et al., Industrial \& Engineering Chemistry Research, 50, 16, (2011).

9. Liu, S.D., et al., Powder Technology, 253, 0, (2014).

10. Zhou, Z.Y., et al., Granular Matter, 16, 5, (2014). 